\title{
An Open-Label Investigation into Drug-Drug Interactions Between Multiple Doses of Daclatasvir and Single-Dose Cyclosporine or Tacrolimus in Healthy Subjects
}

\author{
Marc Bifano - Robert Adamczyk - Carey Hwang • \\ Hamza Kandoussi · Alan Marion • Richard J. Bertz
}

Published online: 21 April 2015

(c) The Author(s) 2015. This article is published with open access at Springerlink.com

\begin{abstract}
Background and Objective Chronic hepatitis $\mathrm{C}$ virus (HCV) infection is a major cause of liver transplantation. Drug-drug interactions (DDIs) with cyclosporine and tacrolimus hindered the use of first-generation protease inhibitors in transplant recipients. The current study investigated DDIs between daclatasvir-a pan-genotypic HCV NS5A inhibitor with clinical efficacy in multiple regimens (including all-oral)—and cyclosporine or tacrolimus in healthy subjects.

Methods Healthy fasted subjects (aged 18-49 years; body mass index $18-32 \mathrm{~kg} / \mathrm{m}^{2}$ ) received single oral doses of cyclosporine $400 \mathrm{mg}$ on days 1 and 9 , and daclatasvir $60 \mathrm{mg}$ once daily on days $4-11$ (group $1, n=14$ ), or a single oral dose of tacrolimus $5 \mathrm{mg}$ on days 1 and 13, and daclatasvir $60 \mathrm{mg}$ once daily on days 8-19 (group 2, $n=14$ ). Blood samples for pharmacokinetic analysis [by liquid chromatography with tandem mass spectrometry (LC-MS/MS)] were collected on days 1 and 9 for cyclosporine (72 h), on days 1 and 13 for tacrolimus (168 h) and on days 8 and 9 (group 1) or on days 12 and 13 (group 2) for daclatasvir $(24 \mathrm{~h})$. Plasma concentrations were determined by validated LC-MS/MS methods.
\end{abstract}

\footnotetext{
M. Bifano · R. Adamczyk $(\bowtie) \cdot$ C. Hwang · R. J. Bertz Bristol-Myers Squibb Research and Development, 311 Pennington Rock Hill Road, Hopewell, NJ 08534, USA e-mail: robert.adamczyk@bms.com
}

H. Kandoussi

Bristol-Myers Squibb Research and Development, Lawrenceville, NJ, USA

\author{
A. Marion \\ ICON Development Solutions, Omaha, NE, USA
}

Results Daclatasvir did not affect the pharmacokinetic parameters of cyclosporine or tacrolimus, and tacrolimus did not affect the pharmacokinetic parameters of daclatasvir. Co-administration of cyclosporine resulted in a $40 \%$ increase in the area under the concentration-time curve of daclatasvir but did not affect its maximum observed concentration.

Conclusion On the basis of these observations in healthy subjects, no clinically relevant DDIs between daclatasvir and cyclosporine or tacrolimus are anticipated in liver transplant recipients infected with HCV; dose adjustments during co-administration are unlikely to be required.

\section{Key Points}

Daclatasvir is a hepatitis C virus NS5A inhibitor with potent pan-genotypic (genotypes 1-6) antiviral activity in vitro.

Daclatasvir did not affect the pharmacokinetic parameters of either cyclosporine or tacrolimus, and tacrolimus did not affect the pharmacokinetic parameters of daclatasvir.

Dose adjustments for daclatasvir, cyclosporine or tacrolimus during co-administration are unlikely to be required.

\section{Introduction}

Decompensated liver disease resulting from hepatitis $\mathrm{C}$ virus (HCV) infection is a leading indication for liver transplantation [1]. Furthermore, post-transplantation HCV 
recurrence is characterized by high levels of HCV replication and accelerated necro-inflammation and fibrosis, significantly lower survival rates relative to those of non$\mathrm{HCV}$ infected transplant recipients, and limited treatment options. Recurrence is ubiquitous if $\mathrm{HCV}$ infection is not eradicated prior to liver transplantation [2,3]. Although pre-transplantation treatment with peginterferon/ribavirin can prevent allograft re-infection if a sustained viral response (SVR) is achieved, treatment is limited by poor tolerability and low efficacy [4]. The low efficacy of this regimen can be increased by the addition of first-generation HCV protease inhibitors (boceprevir and telaprevir); however, early discontinuation and hepatic decompensation rates complicate therapy with therapies that include protease inhibitors [4]. It has also been shown that the addition of the recently approved protease inhibitor simeprevir [5, 6] and the NS5B inhibitor sofosbuvir [7, 8] can improve viral response rates with peginterferon/ ribavirin to a greater degree than boceprevir or telaprevir, and both are well tolerated when administered in conjunction with peginterferon/ribavirin regimens.

In the post-transplantation setting, initiation and maintenance of therapy with peginterferon/ribavirin in patients with recurrent $\mathrm{HCV}$ is complicated by several factors, including clinical characteristics that preclude full-dose therapy [9], dose reductions (in 30-70\% of patients) and premature discontinuations (in $20-40 \%$ of patients) due to adverse events (AEs) [9], low SVR rates (approximately 20\%) [10] and interferon-related immune-mediated allograft dysfunction [11].

Additionally, concomitant use of the current first-generation protease inhibitors in regimens for the treatment of recurrent $\mathrm{HCV}$ in liver transplant recipients is complicated by potentially severe drug-drug interactions (DDIs) with immunosuppressants, such as the calcineurin inhibitors cyclosporine and tacrolimus, for which there is a lifelong requirement. The narrow therapeutic windows of both cyclosporine and tacrolimus-coupled with the facts that both are substrates of cytochrome P450 (CYP) 3A4 and P-glycoprotein (P-gp) [12], and cyclosporine is also an inhibitor of CYP3A4 and P-gp [13]—imply that careful selection of concomitant therapies is required. For example, telaprevir has been shown to cause significant increases in the systemic exposures to both cyclosporine (4.6-fold) and tacrolimus (70-fold) in healthy volunteers [14].

It is clear, therefore, that any direct-acting antiviral agent that is to be used concomitantly with immunosuppressants such as cyclosporine and tacrolimus must have a favourable pharmacokinetic DDI profile in addition to good efficacy and tolerability. It has been shown that no clinically relevant DDIs occur between cyclosporine or tacrolimus and either simeprevir [15] or sofosbuvir [16].
Daclatasvir, a HCV NS5A inhibitor with potent pangenotypic (genotypes 1-6) antiviral activity in vitro [17], has been evaluated in over 5500 patients in combinations with other direct-acting antivirals and peginterferon/ribavirin (data on file; study no. DACL-001). Daclatasvir has a pharmacokinetic profile suitable for once-daily dosing without food restrictions [18], is a substrate of both CYP3A4 [19] and P-gp, and has a low potential for clinically significant DDIs [19-22]. Furthermore, concentrations of unbound daclatasvir are not affected to a clinically significant level by moderate-to-severe hepatic impairment, and dose adjustment is not required for this condition [23].

Only limited clinical data on the use of daclatasvir in transplant recipients concomitantly taking calcineurin inhibitors are available at the current time. Daclatasvir has been used successfully in combination with sofosbuvir as part of a direct-acting antiviral-only regimen without signs of a significant DDI with tacrolimus in a transplant recipient with severe recurrent cholestatic HCV [24]. Daclatasvir has also been used successfully in combination with peginterferon/ribavirin without signs of a significant DDI with cyclosporine in a transplant recipient [25].

The aim of this study was to assess the effects of multiple doses of daclatasvir on the single-dose pharmacokinetics and safety profiles of cyclosporine and tacrolimus, and vice versa.

\section{Methods}

\subsection{Study Design}

This was a phase 1, open-label, single-sequence, two-group study in healthy subjects. The study consisted of a 28-day screening period, a study treatment period and a health status follow-up telephone contact at approximately 7 days post-discharge.

Eligible subjects were admitted to the clinical research facility on day -1 and were required to remain at the facility until discharge. The study duration for subjects in group 1 was approximately 47 days, including the screening period, a 12-day study treatment period and the health status follow-up; subjects in group 1 remained at the research facility for a total of 12 days. The study duration for subjects in group 2 was approximately 55 days, including the screening period, a 20-day study treatment period and the health status follow-up; subjects in group 2 remained at the research facility for a total of 20 days.

Subjects in group 1 received a single oral dose of cyclosporine $400 \mathrm{mg}$ (Neoral ${ }^{\circledR}$; Novartis Pharmaceutical Corporation, East Hanover, NJ, USA) on treatment days 1 and 9; daclatasvir $60 \mathrm{mg}$ once daily (Daklinza ${ }^{\circledR}$; Bristol- 
Myers Squibb, Uxbridge, UK) was administered on days 4-11. The treatment in group 2 consisted of a single oral dose of tacrolimus $5 \mathrm{mg}$ (Prograf ${ }^{\circledR}$; Astellas Pharma, Northbrook, IL, USA) on treatment days 1 and 13; daclatasvir $60 \mathrm{mg}$ once daily was administered on days $8-19$. The dosing schedules differed between the two groups to allow for a wash-out of either cyclosporine or tacrolimus prior to steady-state daclatasvir dosing.

All subjects were required to fast for $10 \mathrm{~h}$ prior to dosing; fasting continued until $4 \mathrm{~h}$ post-dose on days when pharmacokinetic sampling (described below) and clinical laboratory tests were performed (group 1, day 11; group 2, day 19), and until $2 \mathrm{~h}$ post-dose on all other days. With the exception of $240 \mathrm{~mL}$ of water ingested with dosing, subjects refrained from drinking water from $1 \mathrm{~h}$ pre-dose until $1 \mathrm{~h}$ post-dose; water was consumed ad libitum at other times. No concomitant medications (prescription, over-thecounter or herbal) were administered during the study unless they were prescribed by the investigator for the treatment of specific AEs.

This study was conducted in accordance with Good Clinical Practice, as defined by the International Conference on Harmonisation, and in accordance with the ethical principles underlying European Union Directive 2001/20/EC and the US Code of Federal Regulations (CFR), Title 21, Part 50, at two clinical research facilities in the USA (Omaha, NE, and San Antonio, TX) between 27 July 2012 and 16 October 2012. The protocol, protocol amendments and written informed consent form were approved by an Institutional Review Board (IntegReview, Austin, TX, USA). Written informed consent was obtained from each subject at the screening visit prior to the initiation of any study-related procedures.

\subsection{Study Population}

The study population consisted of healthy (as determined by a medical history, physical examination and measurements, vital signs, clinical laboratory tests and 12-lead electrocardiograms) male and female volunteers aged 19-49 years (inclusive) with a body mass index of $18-32 \mathrm{~kg} / \mathrm{m}^{2}$ (inclusive) and a minimum body weight of $60 \mathrm{~kg}$. Female subjects were not allowed to be nursing or pregnant, and had to be using a highly effective method of contraception for at least 1 month prior to dosing; additionally, a negative pregnancy test was required within $24 \mathrm{~h}$ prior to the initial dose of the investigational product.

\subsection{Pharmacokinetic Sample Collection}

Blood samples for the determination of cyclosporine pharmacokinetics were collected on days 1 and 9 [at $0 \mathrm{~h}$ (pre-dose) and at 0.5, 1, 1.5, 2, 4, 8, 12, 16, 24, 48 and
$72 \mathrm{~h}$ post-dose]; samples for tacrolimus pharmacokinetic analysis were collected on days 1 and 13 [at $0 \mathrm{~h}$ (pre-dose) and at $0.5,1,1.5,2,3,4,8,12,16,24,48,72,96,120,144$ and $168 \mathrm{~h}$ post-dose]. Cyclosporine and tacrolimus pharmacokinetic sample collection times varied because of the requirement for a wash-out prior to daclatasvir dosing and differences in the half-life $\left(t_{1 / 2}\right)$ values of cyclosporine and tacrolimus.

Samples for the determination of daclatasvir pharmacokinetics were collected from pre-dose (at $0 \mathrm{~h}$ ) to $0.5,1$, $1.5,2,4,6,8,12,16$ and 24 h post-dose on days 8 and 9 (in group 1) and on days 12 and 13 (in group 2).

Approximately $288 \mathrm{~mL}$ of blood was drawn in total from each subject in group 1 for pharmacokinetic analyses; approximately $333 \mathrm{~mL}$ of blood was drawn from each subject in group 2 .

\subsection{Pharmacokinetic Sample Analyses}

Whole-blood concentrations of cyclosporine and tacrolimus were determined by previously validated liquid chromatography with tandem mass spectrometry (LC-MS/MS) methods by Intertek Pharmaceutical Services (El Dorado Hills, CA, USA) and PPD (Richmond, VA, USA), respectively; daclatasvir plasma concentrations were determined using previously validated LC-MS/MS methods by Tandem Labs (West Trenton, NJ, USA).

Cyclosporine and the internal standard (d4-CSP) were extracted from human whole blood by liquid-liquid extraction using methyl-t-butyl ether (MTBE). After evaporation to dryness and reconstitution, the extracts were analysed by reversed-phase LC-MS/MS coupled with atmospheric pressure ionization (API). Tacrolimus was isolated by liquid-liquid extraction using a mixture of ethyl acetate and hexane. The solvent was evaporated under a nitrogen stream. The final extract was analysed via high-performance liquid chromatography (HPLC) with MS/MS detection. Daclatasvir was extracted from plasma, using liquid-liquid extraction by MTBE; all samples were evaporated under a nitrogen stream. After drying and reconstitution, the extracts were analysed by reversed-phase LC-MS/MS.

Cyclosporine, tacrolimus and daclatasvir chromatography was performed on ACE 5 Phenyl $(50 \times 2.1 \mathrm{~mm}, 5 \mu)$, Thermo Fisher Betasil C18 $(2.1 \mathrm{~mm} \times 50 \mathrm{~mm}, 5 \mu)$ and Fortis Phenyl $(2.1 \mathrm{~mm} \times 50 \mathrm{~mm}, 5 \mu)$ columns, respectively.

The mass analysers for cyclosporine and tacrolimus (API 4000; Applied Biosystems-MDS Sciex) and daclatasvir (API 4000 and API 5000; Applied Biosystems-MDS Sciex) were operated in the positive polarity mode with mass transitions of $\mathrm{m} / \mathrm{z} 1220 / 1203,821 / 768$ and 370/130, respectively; the corresponding limits of detection were $0.1-100,0.25-100$ and $2.0-2000 \mathrm{ng} / \mathrm{mL}$, respectively. 


\subsection{Safety Analyses}

Safety assessments were based on a medical review of $\mathrm{AE}$ reports, physical examinations, electrocardiograms and clinical laboratory tests.

\subsection{Statistical Methods}

\subsubsection{Population Size}

Sample size calculations were based on the precision of estimates and not statistical power concerns. It was considered that 14 subjects in each group were required to ensure that at least 12 subjects in each group completed treatment.

For the assessment of the effect of daclatasvir on cyclosporine or tacrolimus pharmacokinetics, complete data from 12 subjects would provide $90 \%$ confidence intervals (CIs) of the geometric mean ratio (GMR) point estimates of the area under the concentration-time curve (AUC) from time zero to infinite time $\left(\mathrm{AUC}_{\infty}\right)$ and the maximum observed concentration $\left(C_{\max }\right)$ of $87-115 \%$ and $86-117 \%$, respectively, for cyclosporine, and $87-115 \%$ and 79-127\%, respectively, for tacrolimus; complete data from 12 subjects would provide $90 \%$ CIs of the daclatasvir $\mathrm{AUC}_{\infty}$ and $C_{\max }$ GMRs of $91-110 \%$ and 90-112\%, respectively.

\subsubsection{Pharmacokinetic Analyses}

The pharmacokinetic parameters of single-dose cyclosporine and tacrolimus and multiple-dose daclatasvir were derived from blood (cyclosporine and tacrolimus) and plasma (daclatasvir) concentration-time data, using standard non-compartmental methods (WinNonlin ${ }^{\circledR}$ Professional Network Edition, Version 5.2 or higher; Pharsight Corporation, Sunnyvale, CA, USA).

The $C_{\max }$ and the time to reach $C_{\max }\left(T_{\max }\right)$ for singledose cyclosporine or tacrolimus and for multiple-dose daclatasvir were calculated and summarized by treatment. Additional calculated pharmacokinetic parameters of cyclosporine and tacrolimus included the AUC from time zero to the time of the last quantifiable concentration $\left(\mathrm{AUC}_{\mathrm{T}}\right.$; linear up/log down trapezoidal method), $t_{1 / 2}$ and apparent total body clearance $\left(\mathrm{CL}_{\mathrm{T}} / F\right)$. Additional pharmacokinetic parameters of daclatasvir included the minimum observed concentration at the end of the dosing interval $\left(C_{24}\right)$ and AUC during one dosing interval $\left(\mathrm{AUC}_{\mathrm{tau}}\right)$.

The effects of daclatasvir on the pharmacokinetic parameters of cyclosporine and tacrolimus, and vice versa, were assessed by the use of general linear mixed models with fixed effects for treatment and measurements within subject as repeated measures for log-transformed values of $C_{\max }$ and $\mathrm{AUC}_{\mathrm{T}}$ (of cyclosporine and tacrolimus), $\mathrm{AUC}_{\infty}$ (of cyclosporine and tacrolimus), $\mathrm{AUC}_{\text {tau }}$ (of daclatasvir) and $C_{24}$ (of daclatasvir). Point estimates and $90 \%$ CIs of treatment differences on the log scale were exponentiated to obtain estimates of the ratios of the geometric means on the original scale. No adjustments for multiplicity were made.

\subsubsection{Safety Analyses}

All recorded AEs, vital signs, clinical laboratory test results and physical examination findings were listed, tabulated and summarized by treatment group. AEs were categorized by system organ class, preferred term [defined by the Medical Dictionary for Regulatory Activities (MedDRA)], severity and treatment relatedness.

\section{Results}

\subsection{Subject Disposition and Baseline Demographic Characteristics}

A total of 98 subjects provided informed consent to be screened for the study, and 28 subjects $(28.6 \%)$ were enrolled and received study medications. The reasons for screened subjects not receiving study medication included withdrawal of consent $[n=11(11.2 \%)]$, failure to meet study entry criteria $[n=55(56.1 \%)]$ and other reasons $[n=4(4.1 \%)]$.

All subjects received study medication per protocol, completed the study and were included in both the pharmacokinetic and safety evaluation populations. The baseline demographic characteristics of subjects treated in both treatment groups are presented in Table 1.

Table 1 Baseline demographic characteristics of study subjects

\begin{tabular}{lcc}
\hline Characteristic & $\begin{array}{l}\text { Group 1 } \\
(n=14)\end{array}$ & $\begin{array}{l}\text { Group 2 } \\
(n=14)\end{array}$ \\
\hline Age, years & $34.8(7.1)$ & $34.5(8.7)$ \\
Male, $n(\%)$ & $12(85.7)$ & $10(71.4)$ \\
Race, $n(\%)$ & $6(42.9)$ & $10(71.4)$ \\
White & $7(50.0)$ & $4(28.6)$ \\
Black/African American & $1(7.1)$ & 0 \\
Other & $28.5(2.7)$ & $26.3(3.7)$ \\
Body mass index, $\mathrm{kg} / \mathrm{m}^{2}$ &
\end{tabular}

Values are expressed as mean (SD) unless specified otherwise 
3.2 Pharmacokinetics of Cyclosporine and Daclatasvir Administered Alone and in Combination

The concentration-time profiles of single-dose cyclosporine administered alone and in combination with multiple-dose daclatasvir, and multiple-dose daclatasvir administered alone and in combination with single-dose cyclosporine, are presented in Fig. 1a, b, respectively.

The mean concentration-time profiles of single-dose cyclosporine administered alone and in combination with multiple-dose daclatasvir were nearly superimposable (Fig. 1a). Individual measures of cyclosporine pharmacokinetic parameters (Table 2) when administered alone and in combination with daclatasvir were comparable; cyclosporine parameters showed moderate variability, and the elimination was multiphasic. There was no effect of multiple-dose administration of daclatasvir on the single-dose pharmacokinetics of cyclosporine; the $90 \%$ CIs of the GMRs of $C_{\max }$, $\mathrm{AUC}_{\mathrm{T}}$ and $\mathrm{AUC}_{\infty}$ of cyclosporine when concomitantly administered with daclatasvir versus administration alone (Table 3 ) contained 1 and were contained entirely within the accepted boundaries of equivalence ( 0.80 and 1.25$)$.

(a)

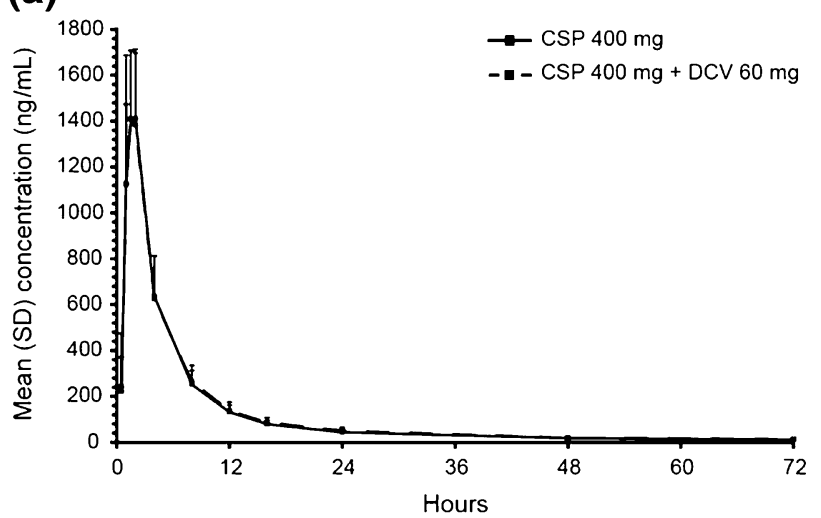

(c)

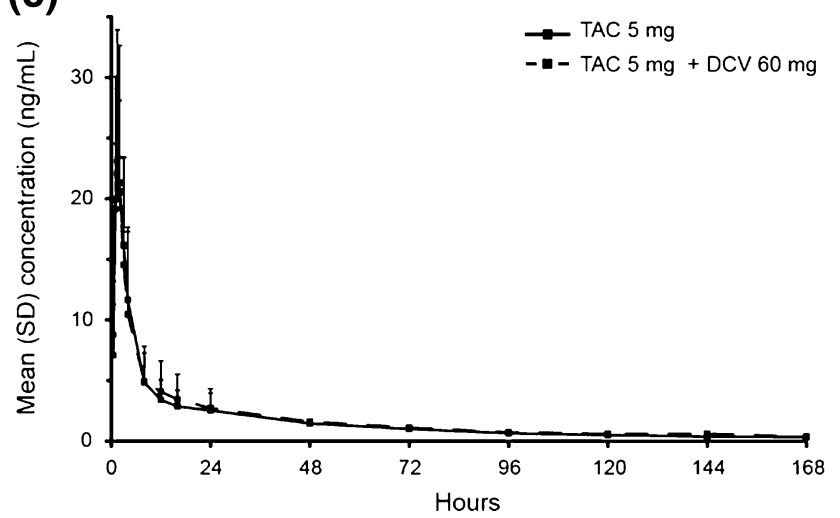

Analyses of pre-dose and trough concentrations indicated that steady-state pharmacokinetics of daclatasvir were achieved within 3 days of dosing (data not shown). When multiple-dose daclatasvir was co-administered with single-dose cyclosporine, the mean peak plasma concentration of daclatasvir was comparable to, but occurred later than, that observed when daclatasvir was administered alone; the rate of elimination appeared to be comparable (Fig. 1b). The geometric mean of the daclatasvir $C_{\max }$ during co-administration with single-dose cyclosporine was comparable to that when daclatasvir was administered alone (Table 2); $\mathrm{AUC}_{\text {tau }}, T_{\max }$ and $C_{24}$ values during coadministration were higher than those during administration of daclatasvir alone and demonstrated moderate variability. Co-administration with single-dose cyclosporine did not significantly affect the $C_{\max }$ of daclatasvir (the $90 \%$ CIs of the GMR were contained within the equivalence boundaries and included 1), although co-administration with cyclosporine increased the daclatasvir $\mathrm{AUC}_{\text {tau }}$ (40\%) and $C_{24}(56 \%)$, with the $90 \%$ CIs of the GMRs of both $\mathrm{AUC}_{\text {tau }}$ and $C_{24}$ being entirely above the upper limit of equivalence (1.25; Table 3$)$.

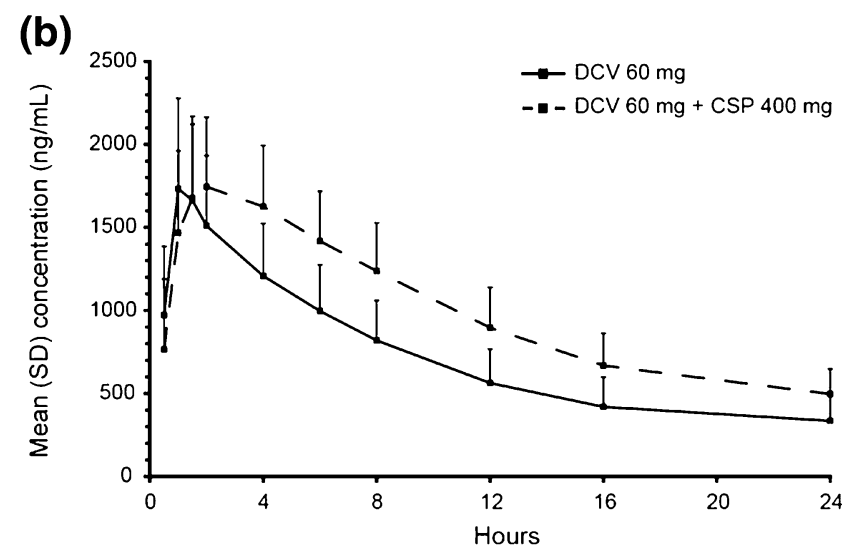

(d)

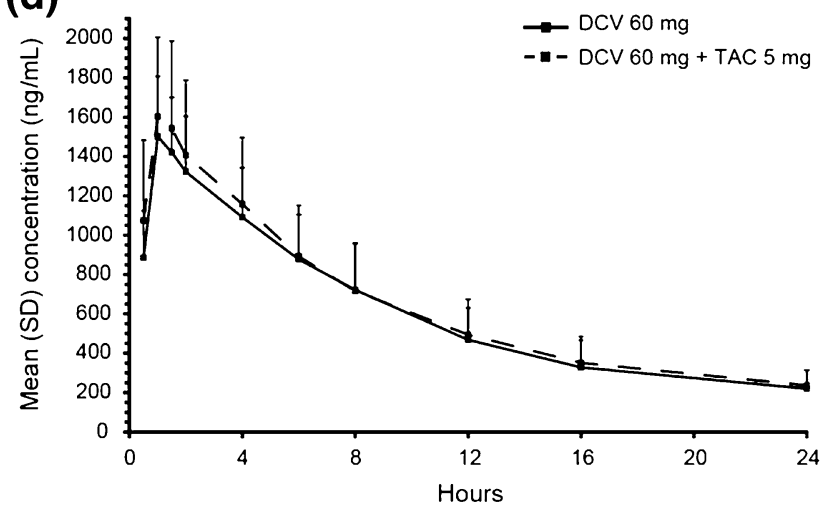

Fig. 1 Concentration-time profiles of (a) cyclosporine (CSP) and (b) daclatasvir (DCV) administered alone and in combination, and (c) tacrolimus (TAC) and (d) DCV administered alone and in combination. SD standard deviation 
Table 2 Pharmacokinetic parameters of daclatasvir administered alone and in combination with either cyclosporine or tacrolimus

\begin{tabular}{|c|c|c|c|c|c|c|c|c|}
\hline \multirow[t]{2}{*}{ Treatment group } & \multicolumn{8}{|c|}{ Pharmacokinetic parameter } \\
\hline & $\begin{array}{l}C_{\max }, \\
\mathrm{ng} / \mathrm{mL}^{\mathrm{a}}\end{array}$ & $\begin{array}{l}\mathrm{AUC}_{\mathrm{T}}, \\
\mathrm{ng} \cdot \mathrm{h} / \mathrm{mL}^{\mathrm{a}}\end{array}$ & $\begin{array}{l}\mathrm{AUC}_{\infty}, \\
\mathrm{ng} \cdot \mathrm{h} / \mathrm{mL}^{\mathrm{a}}\end{array}$ & $\begin{array}{l}\mathrm{AUC}_{\mathrm{tau}} \\
\mathrm{ng} \cdot \mathrm{h} / \mathrm{mL}^{\mathrm{a}}\end{array}$ & $C_{24}, \mathrm{~h}^{\mathrm{a}}$ & $T_{\max }, \mathrm{h}^{\mathrm{b}}$ & $t_{1 / 2}, \mathrm{~h}^{\mathrm{c}}$ & $\mathrm{CL}_{\mathrm{T}} / F, \mathrm{~L} / \mathrm{h}^{\mathrm{a}}$ \\
\hline \multicolumn{9}{|l|}{ Group 1: CSP } \\
\hline CSP alone & $1504(20)$ & $7825(21)$ & $8198(21)$ & ND & ND & $1.5(1.0-2.0)$ & $23.3(3.3)$ & $48.8(23)$ \\
\hline $\mathrm{CSP}+\mathrm{DCV}$ & $1447(20)$ & 7989 (24) & 8405 (24) & ND & ND & $1.5(1.0-2.0)$ & $21.2(5.4)$ & $47.6(25)$ \\
\hline \multicolumn{9}{|l|}{ Group 1: DCV } \\
\hline DCV alone & $1690(31)$ & ND & ND & $16,092(32)$ & $306(44)$ & $1.0(1.0-4.0)$ & ND & ND \\
\hline $\mathrm{DCV}+\mathrm{CSP}$ & $1756(25)$ & ND & ND & $22,587(24)$ & $475(30)$ & $2.0(1.5-4.0)$ & ND & ND \\
\hline \multicolumn{9}{|l|}{ Group 2: TAC } \\
\hline TAC alone & $22.8(28)$ & $225(46)$ & $246(44)$ & ND & ND & $1.5(1.0-2.0)$ & $40.4(8.8)$ & $20.3(72)$ \\
\hline $\mathrm{TAC}+\mathrm{DCV}$ & $24.0(40)$ & $224(59)$ & $245(56)$ & ND & ND & $1.5(0.6-2.0)$ & $38.9(6.5)$ & $20.4(76)$ \\
\hline \multicolumn{9}{|l|}{ Group 2: DCV } \\
\hline DCV alone & $1489(20)$ & ND & ND & $13,786(28)$ & $205(43)$ & $1.0(1.0-1.5)$ & ND & ND \\
\hline $\mathrm{DCV}+\mathrm{TAC}$ & $1578(27)$ & ND & ND & $14,439(30)$ & $226(33)$ & $1.0(0.6-1.5)$ & ND & ND \\
\hline
\end{tabular}

$A U C$ area under the concentration-time curve, $A U C_{\infty}$ AUC from time zero to infinite time, $A U C_{T}$ AUC from zero to the time of the last quantifiable concentration, $A U C_{t a u}$ AUC during one dosing interval, $C_{24}$ minimum observed concentration at the end of the dosing interval, $C L_{T} / F$ apparent total body clearance, $C_{\max }$ maximum observed concentration, $C S P$ cyclosporine, $C V$ coefficient of variation, $D C V$ daclatasvir, $N D$ not determined, $S D$ standard deviation, $t_{1 / 2}$ half-life, $T A C$ tacrolimus, $T_{\max }$ time to reach $C_{\max }$

${ }^{a}$ Geometric mean (CV\%)

b Median (minimum-maximum)

${ }^{c}$ Mean (SD)

Table 3 Statistical analyses of daclatasvir administered alone and in combination with either cyclosporine or tacrolimus

\begin{tabular}{|c|c|c|c|c|c|}
\hline \multirow[t]{2}{*}{ Treatment group } & \multicolumn{5}{|c|}{ Statistical comparison, GMR (90 \% CI) } \\
\hline & $C_{\max }$ & $\mathrm{AUC}_{\mathrm{T}}$ & $\mathrm{AUC}_{\infty}$ & $\mathrm{AUC}_{\mathrm{tau}}$ & $C_{24}$ \\
\hline \multicolumn{6}{|l|}{ Group 1: CSP } \\
\hline CSP + DCV versus CSP alone & $0.96(0.91-1.02)$ & $1.02(0.96-1.08)$ & $1.03(0.97-1.09)$ & ND & ND \\
\hline DCV + CSP versus DCV alone & $1.04(0.94-1.15)$ & ND & ND & $1.40(1.29-1.53)$ & $1.56(1.41-1.71)$ \\
\hline \multicolumn{6}{|l|}{ Group 2: TAC } \\
\hline TAC + DCV versus TAC alone & $1.05(0.90-1.23)$ & $1.00(0.87-1.15)$ & $1.00(0.88-1.13)$ & ND & ND \\
\hline $\mathrm{DCV}+\mathrm{TAC}$ versus DCV alone & $1.07(1.02-1.12)$ & ND & ND & $1.05(1.03-1.07)$ & $1.10(1.03-1.19)$ \\
\hline
\end{tabular}

$A U C$ area under the concentration-time curve, $A U C_{\infty}$ AUC from time zero to infinite time, $A U C_{T}$ AUC from time zero to the time of the last quantifiable concentration, $A U C_{\text {tau }}$ AUC during one dosing interval, $C_{24}$ minimum observed concentration at the end of the dosing interval, $C I$ confidence interval, $C_{\max }$ maximum observed concentration, CSP cyclosporine, DCV daclatasvir, GMR geometric mean ratio, $N D$ not determined, TAC tacrolimus

\subsection{Pharmacokinetics of Tacrolimus and Daclatasvir Administered Alone and in Combination}

The concentration-time profiles of single-dose tacrolimus administered alone and in combination with multiple-dose daclatasvir, and multiple-dose daclatasvir administered alone and in combination with single-dose tacrolimus, are presented in Fig. 1c, d, respectively.

The mean concentration-time profiles of single-dose tacrolimus administered alone and in combination with multiple-dose daclatasvir were comparable (Fig. 1c); the elimination of tacrolimus was slow and multiphasic. The individual measures of the tacrolimus pharmacokinetic parameters (Table 2) when administered alone and in combination with daclatasvir were comparable; the variability in tacrolimus pharmacokinetic parameters was moderate. Concomitant multiple doses of daclatasvir did not affect the single-dose pharmacokinetic of tacrolimus. The $90 \%$ CIs of the GMRs of $C_{\max }, \mathrm{AUC}_{\mathrm{T}}$ and $\mathrm{AUC}_{\infty}$ of tacrolimus when concomitantly administered with daclatasvir, versus administration alone (Table 3), contained 1 and were contained entirely within the accepted boundaries of equivalence.

As in group 1, analyses of pre-dose and trough concentrations indicated that steady-state pharmacokinetics of daclatasvir were achieved within 3 days of dosing 
(data not shown). The plasma concentration-time profiles of multiple-dose daclatasvir in the presence and absence of single-dose tacrolimus were comparable (Fig. 1d), and the elimination phases were comparable. The multiple-dose daclatasvir mean pharmacokinetic parameters were comparable when administered alone or concomitantly with single-dose tacrolimus (Table 2), and measures of variability were also comparable. Concomitant single doses of tacrolimus did not significantly affect the multiple-dose pharmacokinetics of daclatasvir; the $90 \%$ CIs of the GMRs of $C_{\max }, \mathrm{AUC}_{\mathrm{tau}}$ and $C_{24}$ of daclatasvir when concomitantly administered with tacrolimus versus administration alone (Table 3) were contained entirely within the accepted boundaries of equivalence.

\subsection{Safety of Cyclosporine or Tacrolimus Administered Alone or in Combination with Daclatasvir}

There were no serious AEs or AEs leading to discontinuation; all AEs were considered to be of mild intensity and resolved prior to the end of the study, without sequelae. A total of 21 subjects $(75.0 \%)$ reported 69 AEs, of which 58 AEs experienced by 20 subjects were considered related to treatment.

In group 1, 13 of 14 subjects (92.9\%) reported 47 AEs, of which 45 AEs were considered to be related to treatment; more subjects experienced treatment-related AEs during treatment with cyclosporine alone $(8$ subjects, 14 AEs) or in combination with daclatasvir (8 subjects, 16 AEs) than with daclatasvir alone (5 subjects, 15 AEs). Treatment-related AEs in $\geq 2$ subjects were diarrhoea, nausea, headache, sinus congestion and feeling hot (Table 4). Nine of 14 subjects in group 1 reported 13 AEs of feeling hot after treatment including cyclosporine, but none did during treatment with daclatasvir alone; the AEs of feeling hot were not associated with clinically significant elevations in body temperature. The AEs were comparable to those generally observed in patients receiving cyclosporine [13].

In group 2, 8 of 14 subjects $(57.1 \%)$ reported 22 AEs, of which 13 AEs were considered to be related to treatment; an equal number $(n=3)$ of subjects experienced treatment-related AEs during each phase of treatment in this group (tacrolimus alone, $n=3$ AEs; daclatasvir alone, $n=5$ AEs; tacrolimus + daclatasvir, $n=5$ AEs). The treatment-related AEs occurring in $\geq 2$ subjects in group 2 were headache, nausea and constipation (Table 4).

\section{Discussion}

The objective of this study was to assess the effects of multiple doses of daclatasvir on the single-dose pharmacokinetics of cyclosporine or tacrolimus, and the effects of a single dose of either cyclosporine or tacrolimus on the multiple-dose pharmacokinetics of daclatasvir in healthy subjects.

Shared metabolic and distribution pathways often form the basis of DDIs, and substrates and/or inhibitors of CYP3A4 and P-gp are particularly susceptible to such interactions. On the basis that daclatasvir is a substrate of CYP3A4 [19] and a moderate inhibitor of P-gp, cyclosporine is a substrate and inhibitor of both CYP3A4 and P-gp [12, 13], and tacrolimus is a substrate of both CYP3A4 and P-gp [12, 26], it was anticipated that coadministration of daclatasvir with cyclosporine or tacrolimus would not affect the systemic exposures to either cyclosporine or tacrolimus; it was also expected that while tacrolimus would not affect the systemic exposure to daclatasvir, cyclosporine might do so.

The results of this study support these expectations. There was no effect of concomitant multiple doses of daclatasvir on the single-dose exposure to either cyclosporine or tacrolimus. These results suggest that weak-to-moderate inhibition of P-gp by daclatasvir has minimal impacts on the absorption, disposition and excretion of cyclosporine and tacrolimus in vivo. Although a concomitant single dose of tacrolimus did not affect the multiple-dose exposure to daclatasvir, concomitant administration of a single dose of cyclosporine with multiple doses of daclatasvir resulted in increases in the systemic exposure (AUC) and trough concentration $\left(C_{24}\right)$ of daclatasvir; $C_{\max }$ was unaffected. The
Table 4 Treatment-related adverse events occurring in $\geq 2$ subjects in any treatment group phase

CSP cyclosporine,

$D C V$ daclatasvir,

TAC tacrolimus

\begin{tabular}{llllllll}
\hline Preferred term, $n(\%)$ & \multicolumn{2}{l}{ Group 1 treatment phase } & & \multicolumn{3}{l}{ Group 2 treatment phase } \\
\cline { 2 - 3 } & CSP & DCV & CSP + DCV & & TAC & DCV & TAC + DCV \\
\hline Feeling hot & $6(42.9)$ & 0 & $7(50.0)$ & & 0 & 0 & 0 \\
Headache & $3(21.4)$ & $3(21.4)$ & $1(7.1)$ & & 0 & $2(14.3)$ & 0 \\
Diarrhoea & $3(21.4)$ & $1(7.1)$ & $2(14.3)$ & & 0 & 0 & 0 \\
Nausea & $1(7.1)$ & 0 & $3(21.4)$ & & 0 & $1(7.1)$ & $1(7.1)$ \\
Sinus congestion & 0 & $2(14.3)$ & 0 & & 0 & 0 & 0 \\
Constipation & 0 & 0 & 0 & & $2(14.3)$ & 0 & 0
\end{tabular}


increases in the daclatasvir AUC and $C_{24}$ were not considered to be clinically meaningful, on the basis that no exposure-safety relationship was observed during phase 3 dose selection using pharmacokinetic data from multiple studies (daclatasvir dose range 1-100 $\mathrm{mg}$ once daily and $30 \mathrm{mg}$ twice daily) [27]. Among the 694 patients in the population pharmacokinetic model who were within the 4th quartile of steady-state exposures, $13 \%$ of patients had daclatasvir exposures $(>22,000 \mathrm{ng} \cdot \mathrm{h} / \mathrm{mL})$ approximately threefold higher than those observed here, with no unique safety signals observed in this subgroup. Thus, the $40 \%$ increase in exposure observed in this study was well within the range of exposure estimated from the population pharmacokinetic assessment conducted in the phase $2 / 3$ programme using the same data. These data support the use of daclatasvir in transplant recipients and are consistent with observations that when a DDI is observed with daclatasvir, daclatasvir is usually the victim and not the perpetrator of the DDI.

While the results of this study support the concomitant use of daclatasvir with calcineurin inhibitors, it is important to note that only single doses of cyclosporine and tacrolimus were used in this study, to limit the potential for prolonged immunosuppression in healthy subjects. However, the single doses of cyclosporine and tacrolimus used in this study delivered exposures comparable to those observed for the therapeutic ranges of these medications $[13,26]$, and thus these study findings can be extrapolated to the clinical setting of a cyclosporine or tacrolimus multiple dosing scenario. Additionally, a high dose of cyclosporine (400 mg) was used in this study to maximize the probability that if cyclosporine impacted the pharmacokinetics of daclatasvir, such observations would be made.

The use of high doses of cyclosporine and tacrolimus in this study contrasted with those used in the assessment of DDIs with telaprevir [14]. During the assessment of DDIs with telaprevir, in which a DDI was anticipated, single doses of cyclosporine 100 and $10 \mathrm{mg}$ were used during treatment with cyclosporine alone and cyclosporine in addition to telaprevir, respectively; tacrolimus 2 and $0.5 \mathrm{mg}$ were similarly used. During this study, concomitant administration of telaprevir resulted in 4.6 and 70 -fold increases in the exposure to cyclosporine and tacrolimus, respectively, which represented potentially fatal increases in cyclosporine and tacrolimus exposure; approximately fivefold increases in the $t_{1 / 2}$ values of both cyclosporine and tacrolimus were also observed, which indicates the potential for drug accumulation in the clinical setting when multiple doses are used. Furthermore, the degree of the interactions between telaprevir and cyclosporine or tacrolimus [14] were comparable to those observed with human immunodeficiency virus (HIV) protease inhibitors and cyclosporine or tacrolimus, coadministration of which requires significant modifications to both the dosing level and dosing interval of cyclosporine or tacrolimus [28]. In the current study, which revealed no clinically meaningful interactions, the concentrations of cyclosporine and tacrolimus were 40 and 10-fold higher, respectively, than those used by Garg et al. [14].

The use of daclatasvir in transplant recipients has also been reported in two published case reports. Daclatasvir has been used successfully in combination with sofosbuvir as part of a direct-acting antiviral-only regimen without signs of a significant DDI with tacrolimus in a transplant recipient with severe recurrent cholestatic HCV [24], and in combination with peginterferon/ribavirin without signs of a significant DDI with cyclosporine in a transplant recipient [25].

Furthermore, the ability to use daclatasvir without dose adjustments in subjects with hepatic impairment [the unbound daclatasvir AUC values in subjects with Child-Pugh class B (moderate) and class C (severe) hepatic impairment were within $5 \%$ of the unbound daclatasvir AUC values in healthy subjects] [23] offers a simple treatment option for patients approaching the need for liver transplantation (where a rapid reduction in viral load is required) and for patients who have received a liver transplant and require further antiviral therapy. Both populations are considered to have high unmet clinical needs.

\section{Conclusion}

The results of this study indicate that daclatasvir can be used in conjunction with cyclosporine or tacrolimus in transplant recipients, with dose modification unlikely to be required.

Acknowledgments The authors thank the research staff at the participating sites and thank the subjects and their families for their assistance with conducting this study (funded by Bristol-Myers Squibb). Editorial assistance was provided by Andrew Stead of Articulate Science Ltd. and was funded by Bristol-Myers Squibb.

Conflict of interest Marc Bifano was an employee of Bristol-Myers Squibb during the development of this manuscript. Robert Adamczyk, Carey Hwang, Hamza Kandoussi and Richard Bertz are employees of, and may also be stockholders of, Bristol-Myers Squibb. Alan Marion was an employee of ICON Development Solutions during the development of this manuscript.

Open Access This article is distributed under the terms of the Creative Commons Attribution-NonCommercial 4.0 International License (http://creativecommons.org/licenses/by-nc/4.0/), which permits any noncommercial use, distribution, and reproduction in any medium, provided you give appropriate credit to the original author(s) and the source, provide a link to the Creative Commons license, and indicate if changes were made.

\section{References}

1. Verna EC, Brown RS Jr. Hepatitis C virus and liver transplantation. Clin Liver Dis. 2006;10:919-40. 
2. Forman LM, Lewis JD, Berlin JA, Feldman HI, Lucey MR. The association between hepatitis $\mathrm{C}$ infection and survival after orthotopic liver transplantation. Gastroenterology. 2002;122:889-96.

3. Gane EJ, Naoumov NV, Qian KP, et al. A longitudinal analysis of hepatitis $\mathrm{C}$ virus replication following liver transplantation. Gastroenterology. 1996;110:167-77.

4. Verna EC, Terry N, Lukose T, et al. High early response rates with protease inhibitor triple therapy in a multicenter cohort of $\mathrm{HCV}$-infected patients awaiting liver transplantation. Hepatology. 2012;56:218A.

5. Jacobson I, Dore GJ, Foster GR, et al. Simeprevir (TMC435) with peginterferon/ribavirin for chronic HCV genotype-1 infection in treatment-naive patients: results from QUEST-1, a phase III trial [abstract]. J Hepatol. 2013;58(Suppl 1):S574.

6. Manns M, Marcellin P, Fred Poordad FP, et al. Simeprevir (TMC435) with peginterferon/ribavirin for treatment of chronic HCV genotype1 infection in treatment-naive patients: results from QUEST-2, a phase III trial [abstract]. J Hepatol. 2013;58(Suppl 1):S568.

7. Kowdley KV, Lawitz E, Crespo I, et al. Sofosbuvir with pegylated interferon alfa-2a and ribavirin for treatment-naive patients with hepatitis C genotype-1 infection (ATOMIC): an open-label, randomised, multicentre phase 2 trial. Lancet. 2013;381(9883):2100-7.

8. Lawitz E, Lalezari JP, Hassanein T, et al. Sofosbuvir in combination with peginterferon alfa-2a and ribavirin for non-cirrhotic, treatment-naive patients with genotypes 1,2 , and 3 hepatitis $\mathrm{C}$ infection: a randomised, double-blind, phase 2 trial. Lancet Infect Dis. 2013;13(5):401-8.

9. Guillouche P, Feray C. Systematic review: anti-viral therapy of recurrent hepatitis $\mathrm{C}$ after liver transplantation. Aliment Pharmacol Ther. 2011;33:163-74.

10. Bzowej N, Nelson DR, Terrault NA, et al. PHOENIX: a randomized controlled trial of peginterferon alfa-2a plus ribavirin as a prophylactic treatment after liver transplantation for hepatitis $\mathrm{C}$ virus. Liver Transpl. 2011;17:528-38.

11. Selzner N, Guindi M, Renner EL, Berenguer M. Immune-mediated complications of the graft in interferon-treated hepatitis $\mathrm{C}$ positive liver transplant recipients. J Hepatol. 2011;55:207-17.

12. Li DY, Teng RC, Zhu HJ, Fang Y. CYP3A4/5 polymorphisms affect the blood level of cyclosporine and tacrolimus in Chinese renal transplant recipients. Int $\mathbf{J}$ Clin Pharmacol Ther. 2013;51:466-74.

13. Novartis Pharmaceutical Corp. Neoral (cyclosporine) soft gelatin capsules (package insert). 2009.

14. Garg V, Chandorkar G, Farmer HF, Smith F, Alves K, van Heeswijk RP. Effect of telaprevir on the pharmacokinetics of midazolam and digoxin. J Clin Pharmacol. 2012;52:1566-73.

15. Ouwerkerk-Mahadevan S, Simion A, Mortier S, Peeters M, Beumont M. No clinically significant interaction between the investigational HCV protease inhibitor TMC435 and the immunosuppressives cyclosporine and tacrolimus. Hepatology. 2012;64:213A.

16. Mathias A, Cornpropst M, Clemons D, Denning J, Symonds W. No clinically significant pharmacokinetic drug-drug interactions between sofosbuvir (GS-7977) and the immunosuppressants cyclosporine A and tacrolimus in healthy volunteers. Hepatology. 2012;56:A1869.

17. Gao M, Fridell R, Wang C, et al. BMS-766, a novel HCV NS5A inhibitor with enhanced resistance coverage [abstract no. 787]. J Hepatol. 2011;54:S316.

18. Nettles RE, Gao M, Bifano M, et al. Multiple ascending dose study of BMS-790052, a nonstructural protein 5A replication complex inhibitor, in patients infected with hepatitis $\mathrm{C}$ virus genotype 1. Hepatology. 2011;54:1956-65.

19. Bifano M, Sevinsky H, Stonier M, Jiang H, Bertz RJ. Daclatasvir, an HCV NS5A replication complex inhibitor, has minimal effect on pharmacokinetics of midazolam, a sensitive probe for cytochrome P450 3A4 [oral presentation no. O_15]. In: 8th International Workshop on Clinical Pharmacology of Hepatitis Therapy; Cambridge: 2013. Available at http://regist2.virology-education.com/ 2013/8hepcam/docs/21_Bertz.pdf. Accessed July 2014.

20. Bifano M, Sevinsky H, Bedford BR, et al. Coadministration of BMS-790052 and BMS-650032 does not result in a clinically meaningful pharmacokinetic interaction in healthy subjects [abstract]. Hepatology. 2010;52:719A.

21. M, Sevinsky H, Persson A, et al. BMS-790052 has no effect on the pharmacokinetics of a combined oral contraceptive containing ethinyl estradiol and norgestimate in healthy female subjects [poster no. 1340]. AASLD. 2011.

22. Bifano M, Hwang C, Hartstra J, Tiessen RG. Assessment of HIV antiretroviral drug interactions with the HCV NS5A replication complex inhibitor daclatasvir demonstrates a PK profile which supports coadministration with tenofovir, efavirenz and atazanavir/r [poster no. 618]. CROI. 2012.

23. Bifano M, Sevinsky H, Persson A, et al. Single dose pharmacokinetics of BMS-790052 in subjects with hepatic impairment compared with healthy subjects [abstract]. Hepatology. 2011;54(4 Suppl):1005A.

24. Fontana RJ, Hughes EA, Bifano M, et al. Sofosbuvir and daclatasvir combination therapy in a liver transplant recipient with severe recurrent cholestatic hepatitis C. Am J Transplant. 2013;13:1601-5.

25. Fontana RJ, Hughes EA, Appelman H, Hindes R, Dimitrova D, Bifano M. Case report of successful peginterferon, ribavirin, and daclatasvir therapy for recurrent cholestatic hepatitis $\mathrm{C}$ after liver retransplantation. Liver Transpl. 2012;18:1053-9.

26. Astellas Pharma US. Prograf (tacrolimus) capsules (package insert). 2012.

27. Chan P, Tafoya E, Bifano M, et al. Exposure-response analysis of daclatasvir in patients with genotype 1 chronic HCV infection: dose selection for phase 3 clinical trials [abstract no. PK-11]. Rev Antivir Ther Infect Dis. 2012;6:14.

28. Frassetto LA, Browne M, Cheng A, et al. Immunosuppressant pharmacokinetics and dosing modifications in HIV-1 infected liver and kidney transplant recipients. Am J Transplant. 2007;7:2816-20. 